\title{
Stroke prevents exercise-induced gains in bone microstructure but not composition in mice
}

Nicholas J. Hanne ${ }^{1}$, Andrew J. Steward ${ }^{1}$, Marci R. Sessions ${ }^{1}$, Hannah L. Thornburg ${ }^{1}$, Huaxin Sheng ${ }^{2}$, Jacqueline H. Cole ${ }^{1}$

${ }^{1}$ Joint Department of Biomedical Engineering, University of North Carolina, Chapel Hill, NC, and North Carolina State University, Raleigh, NC, USA

${ }^{2}$ Department of Anesthesiology, Duke University Medical Center, Durham, NC, USA

\section{Corresponding Author:}

Jacqueline $\mathrm{H}$. Cole

Joint Department of Biomedical Engineering

University of North Carolina andNorth Carolina State University

911 Oval Drive

Campus Box 7115, Raleigh, NC 27695-7115

jacquecole@ncsu.edu 


\section{ABSTRACT}

Ischemic stroke induces rapid loss in bone mineral density that is up to 13 times greater than during normal aging, leading to a markedly increased risk of fracture. Little is known about skeletal changes following stroke beyond density loss. In this study we use a mild-moderate middle cerebral artery occlusion model to determine the effects of ischemic stroke without bedrest on bone microstructure, dynamic bone formation, and tissue composition. Twentyseven 12-week-old male $\mathrm{C} 57 \mathrm{BI} / 6 \mathrm{~J}$ mice received either a stroke or sham surgery and then either received daily treadmill exercise or remained sedentary for four weeks. All mice were ambulatory immediately following stroke, and limb coordination during treadmill exercise was unaffected by stroke, indicating similar mechanical loading across limbs for both stroke and sham groups. Stroke did not directly detriment microstructure, but exercise only stimulated adaptation in the sham group, not the stroke group, with increased bone volume fraction and trabecular thickness in the sham distal femoral metaphysis. Stroke differentially decreased cortical area in the affected limb relative to the unaffected limb of the distal femoral metaphysis, as well as endosteal bone formation rate in the affected tibial diaphysis. Although exercise failed to improve bone microstructure following stroke, exercise increased mineral-tomatrix content in stroke but not sham. Together, these results show that stroke inhibits exercise-induced changes to femoral microstructure but not tibial composition, even without changes to gait. Similarly, affected-unaffected limb differences in cortical bone structure and bone formation rate in ambulatory mice show that stroke affects bone health even without bedrest.

Keywords: stroke, bone, exercise, microstructure, composition, gait, adaptation 


\section{INTRODUCTION}

2 Stroke is the leading cause of long-term disability in the United States - approximately

37.0 million Americans have had a stroke, and nearly $4 \%$ of the population are projected

4 to have a stroke by 2040 [1]. In addition to cognitive and motor impairments, stroke also

5 severely affects skeletal health, particularly in the paretic limbs, by reducing bone

6 mineral density (BMD) up to 13\% per year compared to $1 \%$ for healthy aging individuals

7 over 60 years of age [2-7]. Reduced BMD and increased susceptibility to falling lead to a

$8 \quad 15 \%$ fracture incidence within 5 years following stroke and a $47 \%$ increased risk of

9 fracture compared to age- and sex-matched controls [8,9]. Traditionally, BMD loss

10 following stroke has been attributed to paresis and bedrest. However, in a study

11 examining BMD a year following severe stroke in completely bed-ridden patients, stroke

12 patients still lost more BMD in their paretic limbs, suggesting stroke impacts skeletal

13 health beyond the effects of mechanical unloading [6]. Aside from BMD loss and

14 increased fracture risk, little is known about the effects of stroke on bone health.

15 Understanding more about how stroke affects other bone measures, particularly at the

16 tissue and cellular levels, is a critical first step for identifying mechanisms underlying

17 bone fragility post-stroke and mitigating bone loss in these patients.

19 Middle cerebral artery occlusion (MCAo) is a well-established technique to induce

20 ischemic stroke in rodents. The MCAo-induced stroke causes ischemia followed by

21 reperfusion damage, mimicking the conditions of the most common type of stroke in

22 human patients $[1,10]$. In this study, we induced a mild to moderate stroke in mice, 
23 ensuring that the animals remained ambulatory after the procedure, to characterize the

24 effect of stroke beyond mechanical disuse effects on bone microstructure, dynamic

25 bone formation, and tissue composition following four weeks of recovery. Since exercise

26 initiated early during stroke recovery is associated with better motor control recovery

27 and BMD maintenance [11-13], mice also performed daily treadmill locomotion. The

28 goals of this study were 1) to characterize the effect of stroke without bedrest on bone

29 parameters beyond $\mathrm{BMD}$, and 2) to determine the effect of moderate daily exercise on

30 stroke-induced bone changes.

\section{METHODS}

33 in vivo Assessments

34 Study Design

35 The protocol for this project was approved by the North Carolina State University

36 Institutional Animal Care and Use Committee. Mice were housed by surgery group (4-5

37 per cage) on a 12-hour diurnal light cycle with access to chow and water ad libitum.

38 Twenty-seven, 12-week-old, male, C57BI/6J mice (The Jackson Laboratory, Bar Harbor,

$39 \mathrm{ME})$ received either a stroke $(n=15)$ or sham $(n=12)$ surgery (Fig. 1$)$. Following surgery,

40 mice were either given daily treadmill exercise $(n=8$ stroke-exercise, $n=6$ sham-

41 exercise) or placed on a stationary treadmill for an equivalent time period ( $n=7$ stroke-

42 sedentary, $\mathrm{n}=6$ sham-sedentary). For four days following surgery, mice were housed

43 individually in cages with wetted food and hydrogel packs, and their health was

44 monitored at least twice a day. Body mass was measured twice a day during the 4-day 
45 acute recovery period and weekly thereafter. On the fifth day, mice were returned to

46 their original cages and group-housed. Stroke recovery and exercise therapy lasted for

47 four weeks following surgery. For dynamic histomorphometry, fluorochrome labels

48 were injected intraperitoneally using a $30 \mathrm{mg} / \mathrm{kg}(0.003 \mathrm{wt} \%)$ concentration at 10 days

49 (alizarin complexone) and 3 days (calcein) prior to sacrifice. After four weeks, mice were

50 euthanized with $\mathrm{CO}_{2}$ asphyxiation followed by cervical dislocation. Left and right femora

51 and tibiae were collected, fixed in $10 \%$ neutral buffered formalin for 18 hours at $4^{\circ} \mathrm{C}$

$52\left(39.2^{\circ} \mathrm{F}\right)$, and then stored in $70 \%$ ethanol at $4^{\circ} \mathrm{C}\left(39.2^{\circ} \mathrm{F}\right)$ until analysis.

\section{Stroke Procedure}

55 After mice were fasted 6-8 hours, ischemic stroke was induced in the right hemisphere

56 using the well-established intraluminal middle cerebral artery occlusion (MCAo) model,

57 in which a thin, silicone-coated 6-0 nylon filament is passed through the external carotid

58 artery (ECA) and internal carotid artery (ICA) to the MCA origin, blocking blood flow to

59 part of the ipsilateral cerebrum (mainly the cortex and basal ganglia) $[10,14,15]$. Mice

60 were anesthetized with isoflurane $(5 \%$ induction, then maintained around $2 \%$

61 throughout the surgery) in a $70: 30$ mixture of $\mathrm{N}_{2}: \mathrm{O}_{2}$ gas. Rectal temperature was

62 maintained at $37^{\circ} \mathrm{C}\left(98.6^{\circ} \mathrm{F}\right)$ throughout the surgery with a heating pad (TCAT-2DF,

63 Physitemp Instruments, LLC, Clifton, NJ). Cerebral blood flow (CBF) was monitored with

64 laser Doppler flowmetry (moorVMS-LDF, Moor Instruments Ltd, Axminster, UK) using a

65 monofilament probe (VP10M200ST, Moor Instruments Ltd). The probe was inserted 
66 through a small skin incision over the right temporal bone, just under the temporalis

67 muscle, and affixed to the skull with a cyanoacrylate glue.

69 The MCAo and sham procedures were performed using aseptic technique. A midline

70 skin incision was made along the neck, and the right common carotid artery (CCA), ECA,

71 and ICA were exposed. For the stroke surgery, the CCA was temporarily ligated, the

72 distal ECA and branches were ligated and cut, and the ICA was then temporarily ligated.

73 At this point, the CCA ligation was temporarily loosened to record a pre-occlusion,

74 baseline value of CBF. The CCA ligation was retightened, and the occluding filament (6-0

75 nylon filament with silicone-coated tip [1-3 mm (0.039-0.12 in) long, 0.22-0.23 mm

76 (0.0087-0.0091 in) in diameter], Doccol Corporation, Redlands, CA) was passed through

77 the stump of the ECA into the ICA until resistance was felt, indicating the occluding

78 filament had reached the MCA origin. The filament was left in place for 30 minutes, and

79 CBF was monitored to ensure an $80 \%$ CBF reduction throughout the occlusion period.

80 Saline was added to the wound to ensure the tissue remained hydrated. After 30

81 minutes, the filament was removed, the ECA ligation was permanently tightened, and

82 the CCA ligation was removed. For the sham surgery, after the neck incision was made

83 and arteries exposed, saline was added to the neck incision, and CBF was monitored for

8430 minutes without inserting the occluding filament. At the end of each surgery, the

85 laser Doppler flowmetry probe was removed, an intra-incisional injection of bupivacaine

86 (2 mg/kg, Marcaine, Hospira, Lake Forest, IL) was administered to the neck incision, and

87 the neck and skull skin incisions were sutured closed. A 4\% lidocaine cream and an 
88 antibiotic ointment were applied to the incision sites, and a subcutaneous injection of

89 carprofen (Rimadyl, Zoetis, Parsippany, NJ) at $5 \mathrm{mg} / \mathrm{kg}(0.0005$ wt \%) was administered.

90

91 Sensorimotor Function

92 Ischemic stroke severity and functional recovery were assessed with a series of scored

93 tests that were summed to form a neurological score, or neuroscore. Each test was

94 scored from 0 to 2 or 0 to 4 , depending on the test. The scores were determined

95 empirically relative to the behavior seen with a sham surgery (score $=0$ ) up to the

96 behavior with severe stroke (score $=48$, the maximum possible score). The scoring

97 system was comprised of four main parts that were modified from similar systems used

98 to assess sensorimotor function during stroke recovery in rats [16,17]: 1) mouse activity

99 level, body symmetry, and gait were scored as the mice were allowed to ambulate freely

100 in an open-top enclosure to assess gross impairments; 2) limb symmetry, circling, and

101 limb placement were also scored during free ambulation to assess motor function; 3 )

102 climbing ability on a vertical metal screen and balance beam walking across a cylindrical

103 rod were scored to determine complex motor function; and 4) sensory function was

104 assessed by gently touching the left (paretic, affected) and right (non-paretic,

105 unaffected) paws, trunk, vibrissae, and face of the mice with a cotton-tip applicator.

106 Neuroscore examinations were performed at 24 hours, 48 hours, 3 days, 4 days, and

107 then weekly following surgery.

108 
109 Stroke recovery was also assessed using a rotarod, or rotating rod, test [15]. Mice were

110 placed on a plastic rod that rotates at a velocity that accelerates from 4 to 40 rotations

111 per minute over 5 minutes (ENV-576M, Med Associates Inc, St. Albans, VT). The test

112 scores motor coordination by timing how long the mouse can walk on the rod without

113 falling off or hugging the rod for three consecutive rotations without attempting to

114 walk. Mice were acclimated to the rotarod test for two days immediately prior to

115 surgery using a constant velocity of 20 rotations per minute. Mice performed

116 accelerating rotarod tests at 48 hours, 4 days, and then weekly following surgery, on the

117 same day as neuroscore testing. During each acclimation and rotarod test day, each

118 mouse attempted the test three times, and the longest time was recorded, up to a

119 maximum of 300 seconds. Mice were allowed 15 min of rest between each attempt.

\section{Treadmill Exercise and Gait Pattern Analysis}

122 Treadmill exercise was performed using a rodent treadmill (Exer 3/6, Columbus

123 Instruments, Columbus, $\mathrm{OH}$ ). Mice in the exercise groups were acclimated to the

124 treadmill for two days prior to surgery at $6 \mathrm{~m} / \mathrm{min}$ (6.56 yds/min) for $10 \mathrm{~min}$. Exercise

125 therapy began at four days after surgery, gradually increasing the protocol for the first

126 two days. On day four, mice were exercised at $5 \mathrm{~m} / \mathrm{min}(5.47 \mathrm{yds} / \mathrm{min})$ for $10 \mathrm{~min}$,

127 followed by $12 \mathrm{~m} / \mathrm{min}$ (13.12 yds/min) for $10 \mathrm{~min}$. On day five, mice exercised at 5

$128 \mathrm{~m} / \mathrm{min}(5.47 \mathrm{yds} / \mathrm{min}$ ) for $5 \mathrm{~min}$, followed by $12 \mathrm{~m} / \mathrm{min}(13.12 \mathrm{yds} / \mathrm{min})$ for $20 \mathrm{~min}$.

129 Every weekday thereafter (5 days/wk) mice performed the full exercise routine of 12

$130 \mathrm{~m} / \mathrm{min}$ (13.12 yds/min) for $30 \mathrm{~min}$. Sedentary group mice were placed on an immobile, 
131 replica treadmill for a matched time period to equalize the stress of animal handling

132 across groups.

133

134 For the exercise groups only, interlimb coordination during treadmill gait was quantified

135 weekly using video analysis. Altered gait in the affected hindlimb could alter the

136 functional tissue strain experienced by bones during locomotion $[18,19]$, which can

137 affect bone formation [20,21]. Gait was captured over 60 s with high-speed video (240

138 frames/sec, HERO4, GoPro Inc, San Mateo, CA) as mice ran on the treadmill at $12 \mathrm{~m} / \mathrm{min}$

139 (13.12 yds/min), and the videos were analyzed using Kinovea (version 0.8, Kinovea Open

140 Source Project). Duty cycle between the hindlimbs and phase dispersion in relation to

141 the left (affected) hindlimb were calculated based on the relative timing of gait pattern

142 events $[18,22,23]$. Duty cycle, the ratio of stance times (paw strike to lift off) across a

143 single gait cycle (paw strike to next paw strike), in the affected hindlimb was calculated

144 relative to the duty cycle in the unaffected hindlimb. Phase dispersion, the relative

145 timing of paw strikes between two limbs within the same gait cycle, was calculated

146 between the affected hindlimb and each of the other limbs: unaffected hindlimb

147 (contralateral), unaffected forelimb (diagonal), and affected forelimb (ipsilateral). All

148 parameters were measured in three sets of five consecutive gait cycles for each weekly

149 treadmill session, and the average parameters were calculated for each week. Video

150 was captured at one day prior to surgery, five days after surgery, and weekly thereafter.

\section{Micro-Computed Tomography}


153 Left and right femora were scanned in $70 \%$ ethanol with micro-computed tomography

154 ( $\mu$ CT80, SCANCO Medical AG, Brüttisellen, Switzerland) using a 10- $\mu \mathrm{m}$ voxel size, $45 \mathrm{kV}$

155 peak X-ray tube potential, $177 \mu \mathrm{A}$ X-ray intensity, and 800-ms integration time. Volumes

156 of interest (VOI) were analyzed in the distal metaphysis and mid-diaphysis. The

157 metaphyseal VOI was defined as $10 \%$ of the total femur length, located proximal to the

158 distal growth plate. The cancellous bone and cortical bone were contoured and analyzed

159 separately in the metaphyseal VOI. Standard cancellous and cortical microstructural

160 parameters were analyzed using the scanner's software (SCANCO v.6.6) [24]. The

161 diaphyseal VOI was defined as $15 \%$ of the total femur length, centered at the midpoint

162 between the distal growth plate and middle of the third trochanter, and standard

163 cortical bone parameters were analyzed.

\section{Dynamic Histomorphometry}

166 Left and right tibiae were cut transversely at the tibiofibular junction under constant

167 irrigation with water using a low-speed precision saw (IsoMet Low Speed Precision

168 Cutter, Buehler, Lake Bluff, IL). The proximal bone segment was saved for Raman

169 spectroscopy, and the distal segment was embedded in methylmethacrylate for

170 dynamic histomorphometry [25]. In the distal segment, approximately $200-\mu m(0.0079-$

171 in) thick transverse sections were cut with the precision saw just distal to the tibiofibular

172 junction, affixed to glass slides with cyanoacrylate glue, then sanded to $10-30 \mu \mathrm{m}$

173 (0.00039-0.0012 in) thickness with increasing grit sandpaper. Two sections from each

174 bone were imaged at 40X on a Zeiss LSM 880 laser scanning microscope with Airyscan 
175 (Carl Zeiss Microscopy, Thornwood, NY). Standard indices of dynamic bone formation

176 were measured on two sections per limb using FIJI (built on ImageJ version 1.51n) and

177 Photoshop (version CC 2018, Adobe Systems Inc., San Jose, CA) [26-28].

178

179 Raman Spectroscopy

180 Cortical bone tissue composition was analyzed in the diaphysis of affected (left) tibiae

181 using Raman spectroscopy. In the proximal bone segment described above,

182 approximately $1-\mathrm{mm}$ (0.39-in) thick sections were cut transversely using the Isomet Low

183 Speed Precision Cutter just proximal to the tibiofibular junction. Sections were affixed to

184 glass slides proximal side down with cyanoacrylate glue, then polished to create a

185 smooth surface. Raman spectra were collected with a 785-nm laser at 50X magnification

186 (Horiba XploRA PLUS, Jobin Yvon, Longjumeau, France). Each cortical section was

187 scanned at the endosteal, mid-cortex, and periosteal edges on the anterior and

188 posterior regions of the cross section (Fig. 2A). Each scan was comprised of a line of 6

189 points spaced $2 \mu \mathrm{m}\left(7.87 \times 10^{-5} \mathrm{in}\right)$ apart, parallel to and 5-10 $\mu \mathrm{m}\left(1.97 \times 10^{-4}-3.94 \times 10^{-4}\right.$ in $)$

190 in from the bone surface. Each point was a 30 -second accumulation in the $800-1800 \mathrm{~cm}^{-}$

1911 range. Baseline correction was performed in LabSpec (v.6.5.1.24, HORIBA, Kyoto,

192 Japan), and spectral analysis was performed with custom code in MATLAB $^{\circledR}$ (R2017, The

193 MathWorks, Natick, MA).

194

195 Raman spectra were normalized relative to the maximum height (intensity) of the 196 phosphate $v_{1}$ band located at $930-980 \mathrm{~cm}^{-1}$, and maximum intensities relative to the 
197 phosphate $v_{1}$ intensity were calculated for the following tissue constituents: summation

198 of proline $\left(830-863 \mathrm{~cm}^{-1}\right)$ and hydroxyproline (wavenumber $864-899 \mathrm{~cm}^{-1}$ ), carbonate $v_{1}$

199 (1055-1090 $\left.\mathrm{cm}^{-1}\right)$, amide I $\left(1616-1720 \mathrm{~cm}^{-1}\right)$, and amide III $\left(1220-1300 \mathrm{~cm}^{-1}\right)[29,30]$ (Fig.

200 2B). Several standard band intensity ratios were calculated [29,31]. Mineral-to-matrix

201 ratios were calculated using phosphate relative to several matrix bands: amide I, amide

202 III, or summed proline and hydroxyproline. Carbonate substitution was calculated as the

203 band intensity ratio of carbonate $v_{1}$ relative to phosphate $v_{1}$, and the carbonate-to-

204 matrix ratio was calculated as carbonate $v_{1}$ relative to amide I. Mineral maturity

205 (crystallinity) was calculated as the inverse of the full width at half maximum (FWHM) of

206 a single-order Gaussian curve fit to the phosphate $v_{1}$ band.

208 Statistical Analysis

209 Statistical models were analyzed in SAS (SAS University Edition v. 9.4, SAS Institute Inc.,

210 Cary, NC) to determine the following: 1) effects of stroke and exercise therapy on

211 neuroscore, rotarod time, and body mass at each timepoint; 2) effect of stroke on gait

212 parameters at each timepoint; 3) effects of stroke and exercise on femoral

213 microstructure, tibial dynamic bone formation, and tibial composition; and 4) whether

214 stroke differentially affects femoral microstructure and tibial bone formation between

215 affected and unaffected limbs.

216

217 For analysis \#1, stroke recovery parameters (neuroscore, rotarod, body mass) were

218 compared between surgery and activity groups at each timepoint using a repeated 
219 measures factorial model (procedure MIXED) with interaction between all terms.

220 Surgery group (sham or stroke) and activity group (sedentary or exercise) were modeled

221 as fixed factors, while timepoint was modeled as a repeated measure within each

222 mouse. The residual variance was modeled assuming compound symmetry covariance.

223 Predicted least-squares means with Tukey-Kramer adjustment for multiple comparisons

224 were used to analyze effect differences between surgery and activity groups, with

225 interaction, within each timepoint (i.e., sham-sedentary vs. stroke-sedentary at Week

226 3). Analysis \#2 used a similar model, except activity group was not included, since gait

227 was only analyzed in the exercise groups (i.e., sham vs. stroke at Week 3).

229 Analyses \#3 and 4 also used a repeated measures factorial model (procedure MIXED).

230 Parameters of femoral microstructure and tibial histomorphometry were analyzed

231 between activity and surgery groups across limb (repeated measure). Tibial composition

232 was only analyzed in the affected tibiae, but scan region (anterior and posterior) was

233 modeled as the repeated measure. Tibial composition in the endosteal, mid-cortex, and

234 periosteal regions were each run as separate models and were not compared. All

235 models used compound symmetry to calculate residual variance. For analysis \#3 - the

236 effect of activity, stroke, and their interaction on femoral and tibial bone parameters -

237 predicted least-squares means were used to perform pairwise differences (i.e., sham-

238 sedentary vs. stroke-sedentary) with Tukey-Kramer adjustments for multiple

239 comparisons. For analysis \#4, least-squares means were used to analyze differences 
240 between limb across surgery group (i.e., affected vs. unaffected limb within stroke

241 group).

242

243 A significance level of 0.05 was used for all analyses. Data are presented as mean \pm

244 standard deviation unless otherwise noted. Data from methods that analyzed affected

245 and unaffected limb bones (i.e., femoral microstructure and tibial histomorphometry)

246 are presented as the mean for both limbs per mouse. Results from Raman spectroscopy

247 are presented as the mean across anterior and posterior quadrants per bone.

249 RESULTS

250 in vivo Assessments

251 Following stroke, all neuroscores were above 0 (sham group score) at all post-stroke

252 timepoints, confirming that the MCAo procedure caused neurological impairment (Fig.

$2533 \mathrm{~A})$. Based on these neuroscores, the severity of the induced strokes was mild to

254 moderate, and the magnitude of impairment varied between individual mice and

255 tended to improve over time (decreasing neuroscore). Exercise did not affect

256 neuroscore at any timepoint $(p=0.66)$, suggesting the treadmill exercise protocol used

257 did not affect stroke recovery. The rotarod test was not a useful metric for assessing

258 motor impairments post-stroke, as it did not capture the same differences as the

259 neuroscore, with no significant effects of stroke $(p=0.88)$ or exercise $(p=0.37)$

260 observed at any timepoint (Fig. 3B). Before surgery, sham and stroke groups had similar

261 body mass $(p=0.38)$, but the stroke group experienced significant mass losses after 
262 ischemic stroke and remained smaller than the sham group at all timepoints post-

263 surgery (Fig. 3C).

264

\section{Gait Pattern Analysis}

266 Limb coordination patterns were quantified in the exercise groups only to avoid

267 confounding the sedentary groups with treadmill activity. The stroke-exercise group was

268 able to perform the daily treadmill regimen beginning four days after stroke. Gait

269 parameters involving the affected (left) hindlimb were mostly similar between stroke

270 and sham groups at every timepoint (Fig. 4). Diagonal phase dispersion was nearly lower

271 in stroke compared to sham groups in Week $1(16 \%, p=0.067)$ and Week $2(3.4 \%, p=$

2720.085 ) (Fig. 4B), but both contralateral (Fig. 4C) and ipsilateral (Fig. 4D) phase

273 dispersions did not differ significantly between stroke and sham groups at any

274 timepoint. The shift in diagonal phase dispersion represents the affected hindlimb paw

275 strike occurring earlier relative to the right forelimb paw lift, which could be a slight limp

276 in the affected hindlimb. However, the duty cycle ratio between the hindlimbs was

277 unaffected by stroke at any timepoint (Fig. 4A), suggesting no differences in hindlimb

278 favorability between sham and stroke groups. Together, these data suggest that limb

279 loading, and thus functional bone tissue strain, during exercise was similar for both

280 groups. One outlier was removed from the sham-exercise group in Week 1, because the

281 limb coordination patterns were irregular, as the mouse was sprinting and pausing

282 excessively and not running at the constant velocity of the treadmill. Several timepoints

283 had videos that could not be analyzed due to poor lighting or camera placement. Except 
284 for Week 2, where only two videos were analyzed for sham-exercise, at least four videos

285 were analyzed per group per timepoint.

287 Micro-Computed Tomography

288 In all VOls, stroke did not affect femoral microstructure (stroke-sedentary vs. sham-

289 sedentary), but exercise improved femoral microstructure in the sham group (sham-

290 exercise vs. sham-sedentary) but not the stroke group (stroke-exercise vs. stroke-

291 sedentary). In the metaphysis, cortical area (Ct.Ar) was nearly greater in sham-exercise

292 than sham-sedentary $(11 \%, p=0.066)$ and was $17 \%$ greater than stroke-exercise $(p=$

2930.0032 ) but was similar between stroke-exercise and stroke-sedentary $(p=0.45)$ (Fig.

294 5A). Similarly, total area (Tt.Ar) was greater in sham-exercise than stroke-exercise (12\%,

$2953.40 \pm 0.19 \mathrm{~mm}^{2}$ vs. $3.03 \pm 0.28 \mathrm{~mm}^{2}, \mathrm{p}=0.046$ ) but was similar between sham-exercise

296 and sham-sedentary (3.12 $\left.\pm 0.06 \mathrm{~mm}^{2}, \mathrm{p}=0.24\right)$, and between stroke-exercise and

297 stroke-sedentary $\left(3.16 \pm 0.14 \mathrm{~mm}^{2}, \mathrm{p}=0.69\right)$. Ct.Ar and Tt.Ar were similar between

298 stroke-sedentary and sham-sedentary ( $p=1.00$ and $p=0.99$, respectively). Bone

299 mineral density (BMD), tissue mineral density (TMD), cortical thickness (Ct.Th), and

300 cortical area fraction (Ct.Ar/Tt.Ar) were not affected by exercise or stroke. In the

301 diaphysis, Ct.Ar was 13-18\% greater in sham-exercise compared to all other groups ( $\mathrm{p}<$

3020.0001 to $p=0.0039)$ but was similar between stroke-exercise and stroke-sedentary $(p=$

$3030.64)$ and between stroke-sedentary and sham-sedentary ( $p=0.97)$ (Fig. 5D). Tt.Ar was

304 nearly greater in sham-exercise than sham-sedentary $(13 \%, 2.28 \pm 0.21$ vs. $2.01 \pm 0.06$

$305 \mathrm{~mm}^{2}, \mathrm{p}=0.085$ ) and was $20 \%$ greater than stroke-exercise (vs. $1.90 \pm 0.14 \mathrm{~mm}^{2}, \mathrm{p}=$ 
3060.0046 ) but was similar between stroke-exercise and stroke-sedentary (vs. $2.03 \pm 0.12$

$\left.307 \mathrm{~mm}^{2}, \mathrm{p}=0.44\right)$ and between stroke-sedentary and sham-sedentary $(\mathrm{p}=1.00) . \mathrm{BMD}$,

308 TMD, Ct.Th (Fig. 5E), and Ct.Ar/Tt.Ar (Fig. 5F) were not affected by exercise or stroke.

310 Similar trends of no stroke effect but inhibited exercise-induced microstructural gains in

311 the stroke group were observed in the cancellous bone of the distal metaphysis.

312 Trabecular thickness (Tb.Th) was greater in sham-exercise than both sham-sedentary

$313(17 \%, p=0.0060)$ and stroke-exercise $(14 \%, p=0.010)$, but stroke-exercise was similar

314 to stroke-sedentary $(p=0.51$ ) (Fig. $5 B)$. Bone volume fraction (BV/TV) was $69 \%$ greater

315 in sham-exercise than sham-sedentary $(p=0.022)$ and nearly greater than stroke-

316 exercise $(38 \%, p=0.10)$, but stroke-exercise was similar to stroke-sedentary $(p=0.49)$

317 (Fig. 5C). Degree of anisotropy (DA) was nearly greater in sham-exercise than sham-

318 sedentary $(11 \%, 1.49 \pm 0.05$ vs. $1.34 \pm 0.06, p=0.089)$ and was $13 \%$ greater than stroke-

319 exercise (vs. $1.33 \pm 0.08, p=0.031$ ), but stroke-exercise was similar to stroke-sedentary

320 (vs. $1.43 \pm 0.11, p=0.24)$. Stroke-sedentary was similar to sham-sedentary for $\mathrm{Tb}$.Th $(p=$

$3210.27), B V / T V(p=0.12)$, and DA $(p=0.47) . B M D, T M D$, trabecular number $(T b . N)$ and

322 separation (Tb.Sp), and connectivity density (Conn.D) were not affected by exercise or

323 stroke. Although stroke did not cause detrimental changes to cancellous microstructure,

324 exercise improved several microstructural parameters in the sham group only,

325 suggesting stroke may inhibit bone anabolism even in ambulatory mice. 
327 Stroke caused affected-unaffected side differences within individual mice (paired

328 comparisons) in some microstructural parameters. In the cortical bone surrounding the

329 distal metaphysis Ct.Ar/Tt.Ar was $1 \%$ smaller ( $p=0.019$, Fig. $5 G$ ) and Ct.Ar was $2 \%$

330 smaller $(p=0.032$, Fig. $5 \mathrm{H})$ in the affected side compared to the unaffected side for the

331 stroke group, but Tt.Ar was similar between stroke affected and unaffected sides $(p=$

332 0.27, Fig. 5I). Cortical BMD was $4 \%$ greater in the affected side compared to the

333 unaffected side for the stroke group (633 \pm 23 vs. $611 \pm 25 \mathrm{mg} / \mathrm{cm}^{3}, \mathrm{p}=0.0018$ ) but

334 similar between the affected and unaffected side for the sham group (634 \pm 17 vs. $632 \pm$

$\left.33516 \mathrm{mg} / \mathrm{cm}^{3}, \mathrm{p}=0.74\right)$. Metaphyseal trabecular parameters and diaphyseal cortical

336 parameters were not significantly different between affected and unaffected sides for

337 the stroke group. Similarly, no affected-unaffected side differences were found in the

338 sham group, except trabecular separation, which was $3 \%$ bigger in the affected than

339 unaffected side ( $238 \pm 34$ vs. $232 \pm 27 \mu \mathrm{m}, \mathrm{p}=0.035)$.

341 Dynamic Histomorphometry

342 Stroke had no effect on dynamic indices of bone formation - mineralizing surface per

343 bone surface (MS/BS), mineral apposition rate (MAR), and bone formation rate (BFR) -

344 on either the periosteal or endosteal surface in the tibial midshaft (Fig. 6). On the

345 endosteal surface, exercise decreased MS/BS by $25 \%$ relative to sedentary $(p=0.0078$,

346 Fig 6A), had no effect on MAR $(p=0.58$, Fig. 6B), and nearly decreased BFR $(14 \%, p=$

347 0.090, Fig. 6C). Although MS/BS did not differ significantly between affected and

348 unaffected tibiae for either stroke or sham groups (Fig. 6D), MAR was $40 \%$ lower in the 
349 affected than the unaffected tibia for the stroke group $(p<0.0001)$ and $30 \%$ lower in

350 the sham group ( $p=0.0045$ ) (Fig. $6 E$ ). Similarly, BFR was $44 \%$ lower in the affected tibia

351 relative to the unaffected tibia for the stroke group $(p=0.0005)$ and nearly lower in the

352 sham group (36\%, $p=0.10$ ) (Fig. 6F). On the periosteal surface, exercise had no effect on

353 MS/BS, MAR, or BFR. The sample size for the sham-exercise group was small $(n=2)$

354 across all of these analyses, because one cage of mice in this group missed the alizarin

355 complexone injection. Therefore, only main effects (exercise, activity) were examined

356 for these parameters, and more samples are needed to confirm the exercise effect

357 observed in the sham-exercise group.

\section{Raman Spectroscopy}

360 Stroke induced minor changes in bone tissue composition in the diaphyseal bone of

361 affected tibiae, while exercise induced more substantial changes, particularly in the

362 stroke group (Fig. 7). Exercise increased phosphate:amide III mineral-to-matrix ratio in

363 stroke-exercise relative to stroke-sedentary by $27 \%$ near the endosteal surface $(p=$

$3640.019)$ and by $25 \%$ in the mid-cortex $(p=0.0070)$ but not in sham-exercise relative to

365 sham-sedentary ( $p=1.00$ endosteal, $p=0.58$ mid-cortex) (Fig. 7A). Stroke nearly

366 decreased phosphate:amide III mineral-to-matrix in stroke-sedentary relative to sham-

367 sedentary near the endosteal surface $(18 \%, p=0.10)$ but not in the midcortex $(p=0.91)$.

368 Exercise also increased the endosteal phosphate:amide I mineral-to-matrix ratio (23\%, $p$

$369=0.079$, Fig. 7C) and the endosteal carbonate-to-matrix ratio (20\%, $p=0.11$, Fig. 7D) in

370 stroke-exercise relative to stroke-sedentary, but not in sham-exercise relative to sham- 
371 sedentary ( $p=0.96$ and $p=0.80$, respectively). Exercise had no effect on the

372 phosphate:(proline+hydroxyproline) mineral-to-matrix ratio (Fig. 7B).

373

374 Stroke decreased carbonate substitution near the periosteal surface by $8 \%$ relative to

375 sham (main effect $p=0.019$, Fig. $7 E$ ) and by $12 \%$ in stroke-sedentary relative to sham-

376 sedentary $(p=0.029)$. Stroke also nearly decreased phosphate:(proline+hydroxyproline)

377 mineral-to-matrix ratio near the endosteal surface $(14 \%, p=0.090$, Fig. $7 \mathrm{~B})$. For

378 carbonate substitution, carbonate-to-matrix ratio, and the phosphate:amide I and

379 phosphate:amide III mineral-to-matrix ratios, the stroke-sedentary group exhibited the

380 most differences relative to the other groups, while stroke-exercise was similar to sham-

381 exercise, suggesting exercise may mitigate the effects of stroke on bone composition.

382 Mineral maturity (crystallinity) was unaffected by stroke or exercise (Fig. 6F).

384 DISCUSSION

385 Ischemic stroke in mice inhibited the anabolic effects of moderate treadmill exercise on

386 femoral bone microstructure. The prevention of exercise-induced adaptations following

387 stroke occurred in the absence of altered gait patterns, and thus despite a normal

388 (sham-level) amount of limb loading. These results directly challenge the current

389 paradigm that stroke effects on bone result primarily from mechanical unloading with

390 bedrest and rather suggest that stroke has direct effects on bone that are independent

391 of disuse effects. In particular, our results show that cortical and trabecular

392 microstructure in the stroke-exercise group was similar to that in both the stroke- 
393 sedentary and sham-sedentary groups, while the sham-exercise group experienced

394 metaphyseal and diaphyseal cortical bone enlargement and trabecular thickening that

395 resulted in a more anisotropic orientation on average. Despite the urgent clinical need

396 to understand the high fracture risk in human stroke patients, to our knowledge this

397 study is only the second one to examine changes to bone tissue in an induced stroke

398 model [32], and the first study in mice. This work reveals that the middle cerebral artery

399 occlusion model of ischemic stroke in mice impacts bone microstructure, bone

400 formation rate, and tissue composition without bedrest; further work with this model

401 can be used to examine mechanisms underlying microstructural changes and inhibited

402 exercise adaptation, which could inform future clinical studies.

404 The effects of stroke on bone were primarily systemic, with similar changes in both

405 affected and unaffected limbs. Stroke did not change gait locomotion patterns in the

406 affected hindlimb compared to sham and, therefore, the stroke and sham groups likely

407 experienced similar functional bone tissue strain, which is known to affect bone

408 remodeling [18-20,33]. Although diagonal phase dispersion was nearly lowered during

409 the first two weeks post-stroke in stroke-exercise relative to sham-exercise, the amount

410 of time spent in stance (duty cycle ratio) was similar between affected and unaffected

411 hindlimbs. Therefore, the functional tissue strain was likely similar as well. Gait patterns

412 were not analyzed in the sedentary groups, since high-speed video had to be captured

413 during treadmill exercise, and even a small amount of exercise could confound the

414 sedentary results. The acute recovery period appears to be a critical window during 
415 which physical activity could prevent skeletal health decline in human stroke patients

416 [34]. Based on a pilot study, we found that the earliest that mice could perform the full

417 treadmill regimen was beginning at four days after stroke. For earlier intervention,

418 alternative physical therapies or exercise-mimicking pharmacological therapies would

419 need to be explored. Future studies could also implement exercise training prior to

420 MCAo, which has been shown to improve functional recovery following stroke [35].

422 Despite mostly systemic effects, stroke did induce differential changes in the affected

$423 \operatorname{limb}$ (relative to the unaffected limb) in cortical bone microstructure and indices of bone

424 formation. Similarly, another MCAo study in 11-week-old male rats that also found no

425 changes in femoral microstructure, reported affected-unaffected limb differences in the

426 stroke group [32]. They found that, in the cortical bone surrounding the distal femoral

427 metaphysis, stroke increased total area (reported as total volume) by $2 \%$ and BMD by

$4285 \%$ in the affected relative to the unaffected side, while we found a $4 \%$ reduction in

429 total area and a similar 3\% increase in BMD. The difference in our total area could be

430 due to stroke interacting with skeletal growth, since 11-week-old rats are at an earlier

431 point in skeletal development than 12-week-old mice. Skeletal growth in C57BI/6J mice

432 slows at 12 weeks and reaches skeletal maturity at 16 weeks, while growth in rats slows

433 around 15 weeks and reaches skeletal maturity at 20 weeks [36-38]. Since animals were

434 ambulatory following stroke in both experiments, these results suggest that stroke,

435 without bedrest, specifically affects the metaphyseal cortical bone envelope. In the

436 tibial diaphysis, stroke differentially decreased mineral apposition rate and bone 
437 formation rate by nearly half relative to the unaffected side. However, a similar

438 unaffected-affected side difference in mineral apposition rate was present in sham, as

439 well. These results are consistent with the MCAo study in rats, which found decreased

440 serum concentration of P1NP, a marker of bone formation, four weeks after stroke [32].

441 Although this outcome was observed in a different bone and site, stroke-induced

442 decreased bone formation rate may contribute to the affected-unaffected limb

443 differences in cortical area fraction observed in the femoral metaphysis.

445 Although exercise did not affect femoral microstructure in the stroke group, it 446 significantly altered tissue composition, an important contributor to bone strength

$447[29,39]$. Along the endosteal surface of the tibial diaphysis of the affected limb, the

448 stroke-exercise group had higher phosphate:amide III and phosphate:amide I mineral-

449 to-matrix ratios and carbonate-to-matrix ratio compared to stroke-sedentary. Since

450 minimal affected-to-unaffected limb differences were observed in other metrics, only

451 affected side tibiae were analyzed. Increased mineral-to-matrix and carbonate-to-matrix

452 ratios indicate stiffer, but potentially more brittle, bone that has not been recently

453 remodeled [29,31]. However, increased mineralization may also be a mechanism by

454 which bone adapts to mechanical load without changing tibial morphology $[20,31,40]$. A

455 study with the same daily treadmill regimen in male $\mathrm{C} 57 \mathrm{BI} / 6 \mathrm{~J}$ mice, initiated at 16

456 weeks-of-age for three weeks, found no changes to tibial morphology but a $15 \%$ higher

457 phosphate:(proline+hydroxyproline) mineral-to-matrix ratio and approximately $10 \%$

458 higher ultimate tensile strain in the tibia with exercise relative to sedentary [40]. 
459 Similarly, a study in young and old rats found that the phosphate:amide I mineral-to-

460 matrix ratio was positively correlated with stiffness but negatively correlated with

461 bending modulus in the femur [31]. Taken together with the dynamic

462 histomorphometry results (lower endosteal mineralizing surface per bone surface with

463 exercise, decreased mineral apposition rate in the stroke-affected limb), the increased

464 tissue mineralization observed in the endosteal surface of stroke-exercise relative to

465 sham-exercise may be compensatory to maintain functional bone tissue strain.

467 Exercise may also impact bone formation, evidenced here by a tendency for reduced

468 mineralizing surface per endosteal bone surface. Treadmill exercise has been shown in

469 other studies to stimulate bone adaptation in mice, although the effect is dependent on

470 the bone envelope, age, sex, and mouse strain - tibiae from younger male mice tend

471 have a greater anabolic response to treadmill exercise than tibiae from older mice past

472 skeletal maturity [33,40-42]. Our results showed no clear anabolic effect with exercise

473 (e.g., no change in bone formation rate and reduced mineralizing surface per surface

474 relative to sham endosteally), but more samples from the sham-exercise group are

475 needed to confirm this observation. However, two recent studies using a similar

476 treadmill regimen in male $\mathrm{C} 57 \mathrm{BI} / 6 \mathrm{~J}$ of similar age also found that exercise had no effect

477 on morphology of the tibial diaphysis or on endosteal bone formation rate, but did

478 increase periosteal bone formation rate in the tibial diaphysis and bone volume fraction

479 in the tibial metaphysis relative to sedentary $[33,41]$. Together, these studies closely

480 mirror our observed exercise-induced increases to trabecular bone volume fraction in 
481 the distal femur and lack of change in dynamic indices of bone formation in the tibia, at

482 least for the endosteal surface.

484 This study revealed that stroke, without bedrest, prevented exercise-induced gains in

485 microstructure, caused affected-unaffected side differences in microstructure and bone

486 formation rate, and affected bone tissue composition. The MCAo mouse model had

487 several advantages over human clinical studies: bone microstructure, dynamic bone

488 formation, and bone composition are difficult to assess in human patients; mice remain

489 ambulatory following stroke, enabling study of the effects of stroke beyond disuse from

490 bedrest; comorbidities preceding ischemic stroke in human patients are not present in

491 mice; and stroke conditions (e.g., location, severity) are tightly controlled. The mouse

492 MCAo model in this study did not cause BMD loss, as seen in human stroke patients, but

493 did cause detriments to microstructure in the affected limb. In the future, the study

494 design could be adapted to try to recapitulate BMD loss seen in human stroke by

495 increasing stroke severity (i.e., occluding the artery for a longer period), increasing the

496 age of the mice, incorporating hindlimb suspension (to mimic bedrest), or extending the

497 endpoint for a longer recovery period. Stroke causes chronic systemic inflammation

$498[43,44]$, declining vascular health [45], and damage to the central nervous system, each

499 of which are known to affect bone remodeling and maintenance $[46,47]$. The MCAo

500 model is an ideal system to explore these interactions and their effects on skeletal

501 health; lifestyle disease models and genetic modifications can be readily generated in

502 mice, the conditions of the stroke are reproducible and easily manipulated, and 
503 different interventions can be implemented to mimic rehabilitation strategies.

504 Improving our understanding of the factors contributing to the reduced skeletal

505 adaptive response following stroke will be essential for developing better strategies to

506 mitigate bone fragility in these patients.

\section{ACKNOWLEDGMENTS}

510 We thank Dr. Ted Bateman and Eric Livingston for micro-CT support; Dr. Roberto Garcia,

511 Dr. Chuanzhen Elaine Zhou, and Dr. Fred Stevie for Raman spectroscopy and sample

512 preparation support; Dr. Eva Johannes for confocal microscopy support; John Biondi and

513 Sophia Tushak for help with dynamic histomorphometry; and Dr. Consuelo Arellano for

514 statistical consulting.

516 This work was performed in part at the Analytical Instrumentation Facility (AIF) at North

517 Carolina State University, which is supported by the State of North Carolina and the

518 National Science Foundation (award number ECCS-1542015). The AIF is a member of the

519 North Carolina Research Triangle Nanotechnology Network (RTNN), a site in the

520 National Nanotechnology Coordinated Infrastructure (NNCl). The authors acknowledge

521 the use of the Cellular and Molecular Imaging Facility (CMIF) at North Carolina State

522 University, which is supported by the State of North Carolina and the National Science 523 Foundation. 


\section{FUNDING}

526 Research reported in this publication was supported by the Eunice Kennedy Shriver

527 National Institute of Child Health and Human Development (NICHD) of the National

528 Institutes of Health (NIH) under award number K12HD073945 and by the American

529 Heart Association (AHA) under award number 7GRNT33710007. The content is solely

530 the responsibility of the authors and does not necessarily represent the official views of

531 the National Institutes of Health.

532 


\section{REFERENCES}

[1] Benjamin, E. J., Muntner, P., Alonso, A., Bittencourt, M. S., Callaway, C. W., Carson, A. P., Chamberlain, A. M., Chang, A. R., Cheng, S., Das, S. R., Delling, F. N., Djousse, L., Elkind, M. S. V., Ferguson, J. F., Fornage, M., Jordan, L. C., Khan, S. S., Kissela, B. M., Knutson, K. L., Kwan, T. W., Lackland, D. T., Lewis, T. T., Lichtman, J. H., Longenecker, C. T., Loop, M. S., Lutsey, P. L., Martin, S. S., Matsushita, K., Moran, A. E., Mussolino, M. E., O’Flaherty, M., Pandey, A., Perak, A. M., Rosamond, W. D., Roth, G. A., Sampson, U. K. A., Satou, G. M., Schroeder, E. B., Shah, S. H., Spartano, N. L., Stokes, A., Tirschwell, D. L., Tsao, C. W., Turakhia, M. P., VanWagner, L. B., Wilkins, J. T., Wong, S. S., Virani, S. S., and American Heart Association Council on Epidemiology and Prevention Statistics Committee and Stroke Statistics Subcommittee, 2019, "Heart Disease and Stroke Statistics-2019 Update: A Report From the American Heart Association," Circulation, 139(10), pp. e56-e528. DOI: 10.1161/CIR.0000000000000659.

[2] Jones, G., Nguyen, T., Sambrook, P., Kelly, P. J., and Eisman, J. A., 1994, “Progressive Loss of Bone in the Femoral Neck in Elderly People: Longitudinal Findings from the Dubbo Osteoporosis Epidemiology Study.," BMJ, 309(6956), pp. 691-695.

[3] Jørgensen, L., Jacobsen, B. K., Wilsgaard, T., and Magnus, J. H., 2000, "Walking after Stroke: Does It Matter? Changes in Bone Mineral Density Within the First 12 Months after Stroke. A Longitudinal Study," Osteoporos Int, 11(5), pp. 381-387. DOI: 10.1007/s001980070103.

[4] Ramnemark, A., Nyberg, L., Lorentzon, R., Englund, U., and Gustafson, Y., 1999, "Progressive Hemiosteoporosis on the Paretic Side and Increased Bone Mineral Density in the Nonparetic Arm the First Year after Severe Stroke," Osteoporos Int, 9(3), pp. 269-275. DOI: 10.1007/s001980050147.

[5] Borschmann, K., Pang, M. Y. C., Iuliano, S., Churilov, L., Brodtmann, A., Ekinci, E. I., and Bernhardt, J., 2015, "Changes to Volumetric Bone Mineral Density and Bone Strength after Stroke: A Prospective Study," Int J Stroke, 10(3), pp. 396-399. DOI: 10.1111/ijs.12228.

[6] Beaupré, G. S., and Lew, H. L., 2006, “Bone-Density Changes After Stroke," American Journal of Physical Medicine \& Rehabilitation, 85(5), pp. 464-472. DOI: 10.1097/01.phm.0000214275.69286.7a.

[7] Jørgensen, L., and Jacobsen, B. K., 2001, "Functional Status of the Paretic Arm Affects the Loss of Bone Mineral in the Proximal Humerus after Stroke: A 1-Year Prospective Study," Calcif. Tissue Int., 68(1), pp. 11-15. DOI: $10.1007 / \mathrm{s} 002230001165$.

[8] Ramnemark, A., Nyberg, L., Borssén, B., Olsson, T., and Gustafson, Y., 1998, "Fractures after Stroke," Osteoporos Int, 8(1), pp. 92-95. DOI: 10.1007/s001980050053.

[9] Kapral, M. K., Fang, J., Alibhai, S. M. H., Cram, P., Cheung, A. M., Casaubon, L. K., Prager, M., Stamplecoski, M., Rashkovan, B., and Austin, P. C., 2017, "Risk of Fractures after Stroke," Neurology, 88(1), pp. 57-64. DOI: 10.1212/WNL.0000000000003457. 
[10] Longa, E. Z., Weinstein, P. R., Carlson, S., and Cummins, R., 1989, "Reversible Middle Cerebral Artery Occlusion without Craniectomy in Rats.," Stroke, 20(1), pp. 84-91. DOI: 10.1161/01.STR.20.1.84.

[11] Peppen, R. P. V., Kwakkel, G., Wood-Dauphinee, S., Hendriks, H. J., Wees, P. J. V. der, and Dekker, J., 2004, "The Impact of Physical Therapy on Functional Outcomes after Stroke: What's the Evidence?," Clin Rehabil, 18(8), pp. 833-862. DOI: 10.1191/0269215504cr843oa.

[12] Bernhardt, J., English, C., Johnson, L., and Cumming, T. B., 2015, "Early Mobilization After Stroke Early Adoption but Limited Evidence," Stroke, 46(4), pp. 1141-1146. DOI: 10.1161/STROKEAHA.114.007434.

[13] Borschmann, K., Iuliano, S., Ghasem-Zadeh, A., Churilov, L., Pang, M. Y. C., and Bernhardt, J., 2018, "Upright Activity and Higher Motor Function May Preserve Bone Mineral Density within 6 Months of Stroke: A Longitudinal Study," Arch Osteoporos, 13(1), p. 5. DOI: 10.1007/s11657-017-0414-4.

[14] Park, S.-Y., Marasini, S., Kim, G.-H., Ku, T., Choi, C., Park, M.-Y., Kim, E.-H., Lee, Y.D., Suh-Kim, H., and Kim, S.-S., 2014, "A Method for Generate a Mouse Model of Stroke: Evaluation of Parameters for Blood Flow, Behavior, and Survival," Exp Neurobiol, 23(1), pp. 104-114. DOI: 10.5607/en.2014.23.1.104.

[15] Bouët, V., Freret, T., Toutain, J., Divoux, D., Boulouard, M., and Schumann-Bard, P., 2007, "Sensorimotor and Cognitive Deficits after Transient Middle Cerebral Artery Occlusion in the Mouse," Experimental Neurology, 203(2), pp. 555-567. DOI: 10.1016/j.expneurol.2006.09.006.

[16] Bederson, J. B., Pitts, L. H., Tsuji, M., Nishimura, M. C., Davis, R. L., and Bartkowski, H., 1986, "Rat Middle Cerebral Artery Occlusion: Evaluation of the Model and Development of a Neurologic Examination," Stroke, 17(3), pp. 472-476.

[17] Reglődi, D., Tamás, A., and Lengvári, I., 2003, "Examination of Sensorimotor Performance Following Middle Cerebral Artery Occlusion in Rats," Brain Research Bulletin, 59(6), pp. 459-466. DOI: 10.1016/S0361-9230(02)00962-0.

[18] Prasad, J., Wiater, B. P., Nork, S. E., Bain, S. D., and Gross, T. S., 2010, "Characterizing Gait Induced Normal Strains in a Murine Tibia Cortical Bone Defect Model," J. Biomech., 43(14), pp. 2765-2770. DOI: 10.1016/j.jbiomech.2010.06.030.

[19] Hurwitz, D. E., Sumner, D. R., Andriacchi, T. P., and Sugar, D. A., 1998, "Dynamic Knee Loads during Gait Predict Proximal Tibial Bone Distribution," J. Biomech., 31(5), pp. 423-430. DOI: 10.1016/S0021-9290(98)00028-1.

[20] Frost, H. M., 2003, “Bone's Mechanostat: A 2003 Update,” Anat. Rec., 275A(2), pp. 1081-1101. DOI: 10.1002/ar.a.10119.

[21] Ellman, R., Spatz, J., Cloutier, A., Palme, R., Christiansen, B. A., and Bouxsein, M. L., 2013, "Partial Reductions in Mechanical Loading Yield Proportional Changes in Bone Density, Bone Architecture, and Muscle Mass," J. Bone. Miner. Res., 28(4), pp. 875-885. DOI: 10.1002/jbmr.1814.

[22] Leblond, H., L'Espérance, M., Orsal, D., and Rossignol, S., 2003, "Treadmill Locomotion in the Intact and Spinal Mouse," J. Neurosci., 23(36), pp. 11411-11419.

[23] Kloos, A. D., Fisher, L. C., Detloff, M. R., Hassenzahl, D. L., and Basso, D. M., 2005, "Stepwise Motor and All-or-None Sensory Recovery Is Associated with Nonlinear 
Sparing after Incremental Spinal Cord Injury in Rats," Exp. Neurol., 191(2), pp. 251265. DOI: 10.1016/j.expneurol.2004.09.016.

[24] Bouxsein, M. L., Boyd, S. K., Christiansen, B. A., Guldberg, R. E., Jepsen, K. J., and Müller, R., 2010, "Guidelines for Assessment of Bone Microstructure in Rodents Using Micro-Computed Tomography," J Bone Miner Res, 25(7), pp. 1468-1486. DOI: 10.1002/jbmr.141.

[25] Smith, L., Bigelow, E. M. R., and Jepsen, K. J., 2013, "Systematic Evaluation of Skeletal Mechanical Function," Curr Protoc Mouse Biol, 3, pp. 39-67. DOI: 10.1002/9780470942390.mo130027.

[26] Dempster, D. W., Compston, J. E., Drezner, M. K., Glorieux, F. H., Kanis, J. A., Malluche, H., Meunier, P. J., Ott, S. M., Recker, R. R., and Parfitt, A. M., 2013, "Standardized Nomenclature, Symbols, and Units for Bone Histomorphometry: A 2012 Update of the Report of the ASBMR Histomorphometry Nomenclature Committee," J Bone Miner Res, 28(1), pp. 2-17. DOI: 10.1002/jbmr.1805.

[27] Egan, K. P., Brennan, T. A., and Pignolo, R. J., 2012, "Bone Histomorphometry Using Free and Commonly Available Software," Histopathology, 61(6), pp. 11681173. DOI: 10.1111/j.1365-2559.2012.04333.x.

[28] Schindelin, J., Arganda-Carreras, I., Frise, E., Kaynig, V., Longair, M., Pietzsch, T., Preibisch, S., Rueden, C., Saalfeld, S., Schmid, B., Tinevez, J.-Y., White, D. J., Hartenstein, V., Eliceiri, K., Tomancak, P., and Cardona, A., 2012, "Fiji: An OpenSource Platform for Biological-Image Analysis," Nature Methods, 9(7), pp. 676-682. DOI: 10.1038/nmeth.2019.

[29] Morris, M. D., and Mandair, G. S., 2011, "Raman Assessment of Bone Quality," Clin Orthop Relat Res, 469(8), pp. 2160-2169. DOI: 10.1007/s11999-010-1692-y.

[30] Gamsjaeger, S., Masic, A., Roschger, P., Kazanci, M., Dunlop, J. W. C., Klaushofer, K., Paschalis, E. P., and Fratzl, P., 2010, "Cortical Bone Composition and Orientation as a Function of Animal and Tissue Age in Mice by Raman Spectroscopy," Bone, 47(2), pp. 392-399. DOI: 10.1016/j.bone.2010.04.608.

[31] Akkus, O., Adar, F., and Schaffler, M. B., 2004, “Age-Related Changes in Physicochemical Properties of Mineral Crystals Are Related to Impaired Mechanical Function of Cortical Bone," Bone, 34(3), pp. 443-453. DOI: 10.1016/j.bone.2003.11.003.

[32] Borschmann, K. N., Rewell, S. S., Iuliano, S., Ghasem-Zadeh, A., Davey, R. A., Ho, H., Skeers, P. N., Bernhardt, J., and Howells, D. W., 2017, "Reduced Bone Formation Markers, and Altered Trabecular and Cortical Bone Mineral Densities of Non-Paretic Femurs Observed in Rats with Ischemic Stroke: A Randomized Controlled Pilot Study," PLoS One; San Francisco, 12(3), p. e0172889. DOI: http://dx.doi.org.prox.lib.ncsu.edu/10.1371/journal.pone.0172889.

[33] Berman, A. G., Hinton, M. J., and Wallace, J. M., 2019, "Treadmill Running and Targeted Tibial Loading Differentially Improve Bone Mass in Mice," Bone Rep, 10. DOI: 10.1016/j.bonr.2019.100195.

[34] Borschmann, K., Pang, M. Y. C., Bernhardt, J., and luliano-Burns, S., 2012, "Stepping towards Prevention of Bone Loss after Stroke: A Systematic Review of the 
664

665

666

667

668

669

670

671

672

673

674

675

676

677

678

679

680

681

682

683

684

685

686

687

688

689

690

691

692

693

694

695

696

697

698

699

700

701

702

703

704

705

706

Skeletal Effects of Physical Activity after Stroke," International Journal of Stroke, 7(4), pp. 330-335. DOI: 10.1111/j.1747-4949.2011.00645.x.

[35] Gertz, K., Priller, J., Kronenberg, G., Fink, K. B., Winter, B., Schröck, H., Ji, S., Milosevic, M., Harms, C., Böhm, M., Dirnagl, U., Laufs, U., and Endres, M., 2006, "Physical Activity Improves Long-Term Stroke Outcome via Endothelial Nitric Oxide Synthase-Dependent Augmentation of Neovascularization and Cerebral Blood Flow," Circ. Res., 99(10), pp. 1132-1140. DOI:

10.1161/01.RES.0000250175.14861.77.

[36] Somerville, J. M., Aspden, R. M., Armour, K. E., Armour, K. J., and Reid, D. M., 2004, "Growth of C57BL/6 Mice and the Material and Mechanical Properties of Cortical Bone from the Tibia," Calcif. Tissue Int., 74(5), pp. 469-475. DOI: 10.1007/s00223-003-0101-x.

[37] Hughes, P. C., and Tanner, J. M., 1970, "The Assessment of Skeletal Maturity in the Growing Rat.," J Anat, 106(Pt 2), pp. 371-402.

[38] Horton, J. A., Bariteau, J. T., Loomis, R. M., Strauss, J. A., and Damron, T. A., 2008, "Ontogeny of Skeletal Maturation in the Juvenile Rat," The Anatomical Record, 291(3), pp. 283-292. DOI: 10.1002/ar.20650.

[39] van der Meulen, M. C. H., Jepsen, K. J., and Mikić, B., 2001, “Understanding Bone Strength: Size Isn't Everything," Bone, 29(2), pp. 101-104. DOI: 10.1016/S87563282(01)00491-4.

[40] Kohn, D. H., Sahar, N. D., Wallace, J. M., Golcuk, K., and Morris, M. D., 2008, "Exercise Alters Mineral and Matrix Composition in the Absence of Adding New Bone," Cells Tissues Organs; Basel, 189(1-4), pp. 33-7.

[41] Gardinier, J. D., Rostami, N., Juliano, L., and Zhang, C., 2018, "Bone Adaptation in Response to Treadmill Exercise in Young and Adult Mice," Bone Rep, 8, pp. 29-37. DOI: 10.1016/j.bonr.2018.01.003.

[42] Wallace, J. M., Rajachar, R. M., Allen, M. R., Bloomfield, S. A., Robey, P. G., Young, M. F., and Kohn, D. H., 2007, "Exercise-Induced Changes in the Cortical Bone of Growing Mice Are Bone- and Gender-Specific," Bone, 40(4), pp. 1120-1127. DOI: 10.1016/j.bone.2006.12.002.

[43] Fassbender, K., Rossol, S., Kammer, T., Daffertshofer, M., Wirth, S., Dollman, M., and Hennerici, M., 1994, "Proinflammatory Cytokines in Serum of Patients with Acute Cerebral Ischemia: Kinetics of Secretion and Relation to the Extent of Brain Damage and Outcome of Disease," Journal of the neurological sciences, 122(2), pp. 135-139.

[44] Tuttolomondo, A., Di Raimondo, D., di Sciacca, R., Pinto, A., and Licata, G., 2008, "Inflammatory Cytokines in Acute Ischemic Stroke," Current Pharmaceutical Design; Schiphol, 14(33), pp. 3574-89. DOI: http://dx.doi.org.prox.lib.ncsu.edu/10.2174/138161208786848739.

[45] Pang, M. Y. C., Yang, F. Z. H., and Jones, A. Y. M., 2013, "Vascular Elasticity and Grip Strength Are Associated With Bone Health of the Hemiparetic Radius in People With Chronic Stroke: Implications for Rehabilitation," PHYS THER, 93(6), pp. 774785. DOI: 10.2522/ptj.20120378. 
bioRxiv preprint doi: https://doi.org/101101/708388; this version posted July 19,2019 . The copyright holder for this preprint (which was not certified by peer review) is the author/funder, who has granted bioRxiv a license to display the preprint in perpetuity. It is made available under aCC-BY 4.0 International license.

707 [46] Stegen, S., and Carmeliet, G., 2018, "The Skeletal Vascular System - Breathing 708 Life into Bone Tissue," Bone, 115, pp. 50-58. DOI: 10.1016/j.bone.2017.08.022.

709 [47] Wong, I. P. L., Zengin, A., Herzog, H., and Baldock, P. A., 2008, "Central 710 Regulation of Bone Mass," Seminars in Cell \& Developmental Biology, 19(5), pp.

711 452-458. DOI: 10.1016/j.semcdb.2008.08.001.

712 


\section{Figure Captions}

Figure 1. Experimental design: Twenty-seven $\mathrm{C} 57 \mathrm{BI} / 6 \mathrm{~J}$ mice received either a sham or stroke surgery at 12 weeks of age. Mice were further divided into either a moving treadmill exercise group or a stationary treadmill sedentary group. After four weeks of recovery, mice were sacrificed, and their femora and tibiae were collected for analysis.

Figure 2. A) In cortical sections of the affected (left) tibial diaphysis, Raman spectra were collected at 6 equallyspaced regions, along a 10- $\mu \mathrm{m}$ long line on the anterior and posterior sides at three locations: endosteal edge, mid-cortex, and periosteal edge. B) Raman spectra were normalized to the phosphate phosphate $v_{1}$ band intensity, and band intensity ratios were calculated: phosphate $v_{1}$ /(proline+hydroxyproline), phosphate $v_{1}$ /carbonate $v_{1}$ (carbonate substitution), phosphate $v_{1}$ /amide I, phosphate $v_{1}$ /amide III, and carbonate $v_{1}$ /amide I. Crystallinity was measured as the inverse of the full width at half-maximum (FWHM) of the phosphate $v_{1}$ band.

Figure 3. Stroke severity and recovery were assessed with A) scored tests of sensorimotor function (neuroscore), B) length of time spent on an accelerating rod, and C) body mass. Data presented as estimated least-squares means $\pm 95 \%$ confidence intervals. ${ }^{*} p<0.05$ stroke vs. sham within timepoint.

Figure 4. Limb coordination parameters involving the affected left hindlimb during treadmill locomotion were all unaffected by stroke: A) duty cycle ratio between affected left hindlimb (LHL) and unaffected right hindlimb (RHL), B) diagonal phase dispersion, $\mathrm{C}$ ) contralateral phase dispersion, and D) ipsilateral phase dispersion. $a^{\prime}: p<0.1$ shamexercise vs. stroke-exercise within timepoint

Figure 5. Femoral microstructure was assessed for group differences in the distal metaphysis (A-C) and mid-diaphysis (D-F) and for affected-unaffected limb differences in the distal metaphysis (G-I). In the metaphysis, exercise in sham, but not stroke, caused increased A) cortical area, B) trabecular thickness, and C) bone volume fraction. In the diaphysis, D) Ct.Ar also increased with sham-exercise relative to sham-sedentary and stroke-exercise; E) cortical thickness and F) cortical area per total area were unaffected by stroke or exercise. In the metaphysis, affected-to-unaffected limb differences were observed in stroke but not sham groups for G) Ct.Ar/Tt.Ar and H) Ct.Ar but not I) Tt.Ar. $a: p<0.05$ sham-exercise vs. stroke exercise. $a^{\prime}: p<0.1$ sham-exercise vs. stroke exercise. $b: p<0.05$ sham-exercise vs. sham-sedentary. $b^{\prime}: p<0.1$ sham-exercise vs. sham-sedentary. $c: p<0.05$ vs. unaffected side within surgery group.

Figure 6. Dynamic cortical bone formation was assessed in the tibial diaphysis with dynamic histomorphometry. No differences were found on the periosteal surface. On the endosteal surface, exercise decreased (relative to sedentary) A) mineralizing surface per bone surface (MS/BS) but not $B$ ) mineral apposition rate (MAR) or C) bone formation rate (BFR). For affected (Aff) vs. unaffected (Un) limb differences, neither 
sham nor stroke group showed D) differences for MS/BS, but E) both sham and stroke had decreased MAR in the affected limbs, and F) stroke decreased BFR in the affected limb. (A-C) Data presented as estimated least-squares mean $\pm 95 \%$ confidence interval. c: $p<0.05$ vs. unaffected side within surgery group. $d: p<0.05$ exercise vs. sedentary (main effect).

Figure 7. Bone tissue composition assessed in the endosteal, mid-cortex, and periosteal regions of the affected tibial diaphysis revealed significant changes with exercise relative to sedentary and minor changes with stroke relative to sham: A) phosphate $v_{1}$ /amide III, B) phosphate $v_{1}$ /(proline+hydroxyproline), C) phosphate $v_{1}$ /amide I, D) carbonate $v_{1}$ /amide I, E) carbonate substitution (carbonate $v_{1}$ /phosphate $v_{1}$ ), and F) mineral crystallinity (phosphate $v_{1}$ full width at half maximum, FWHM). e: $p<0.05$ strokeexercise vs. stroke-sedentary. $f: p<0.05$ stroke-sedentary vs. sham-sedentary. $g$ : $p<$ 0.05 stroke vs sham (main effect). 


\section{Figure 1}

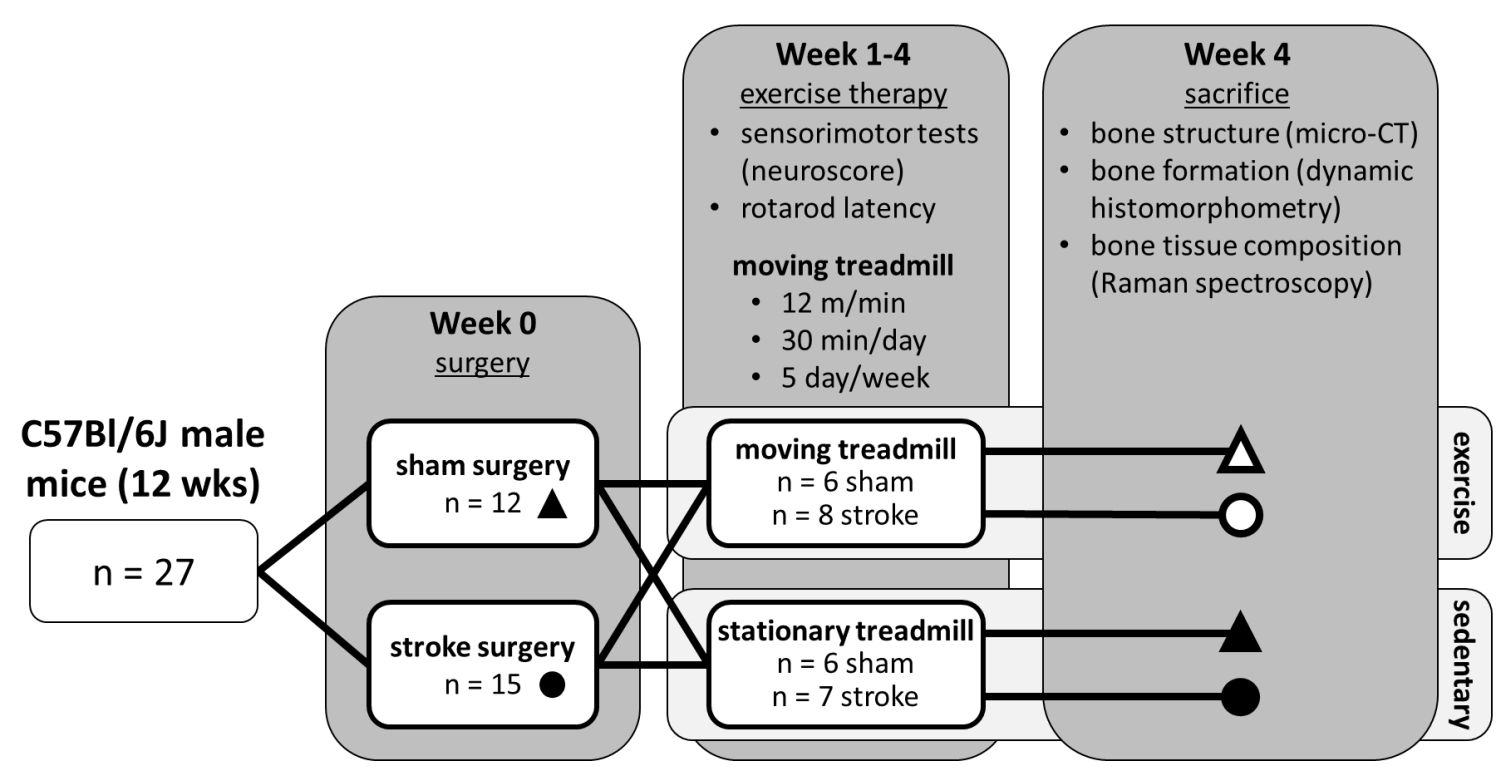




\section{Figure 2}

A
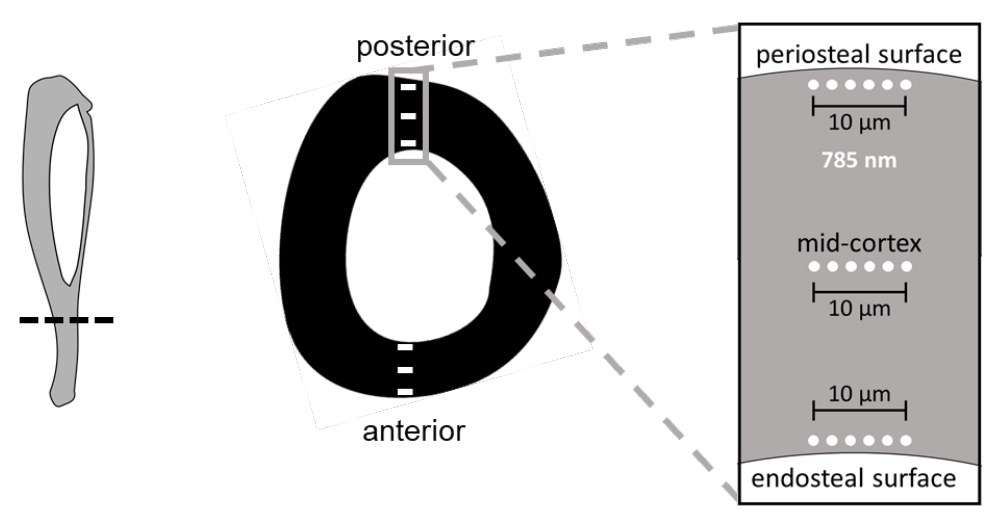

B

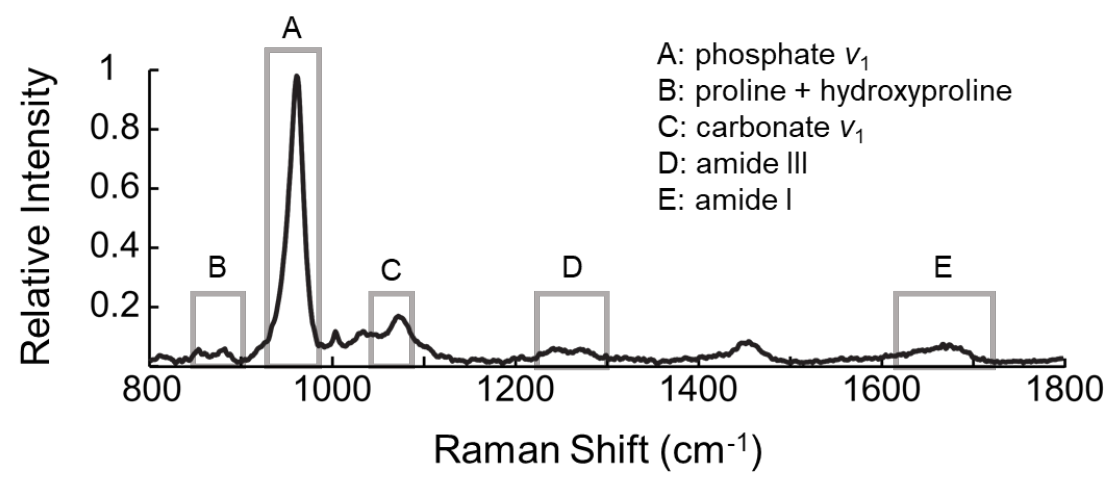




\section{Figure 3}
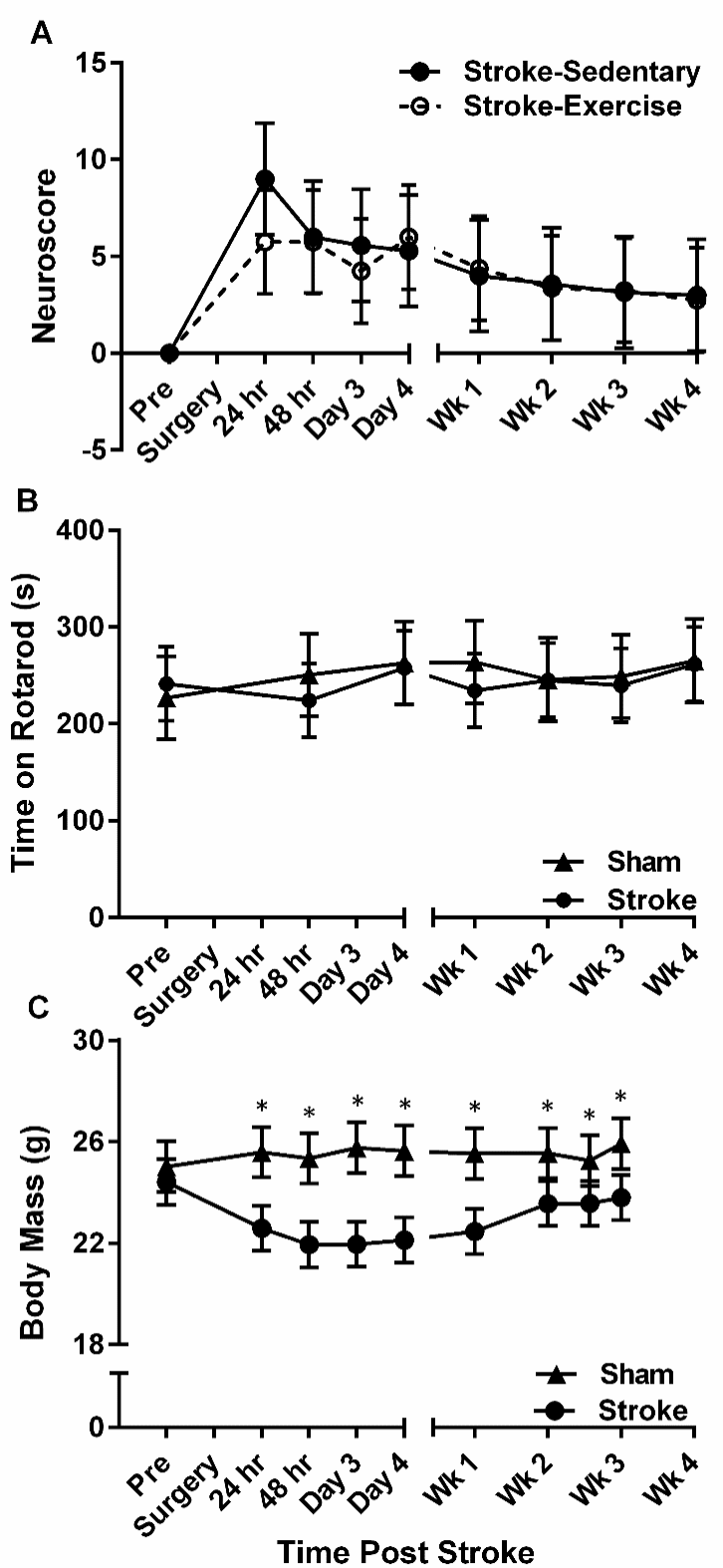


\section{Figure 4}
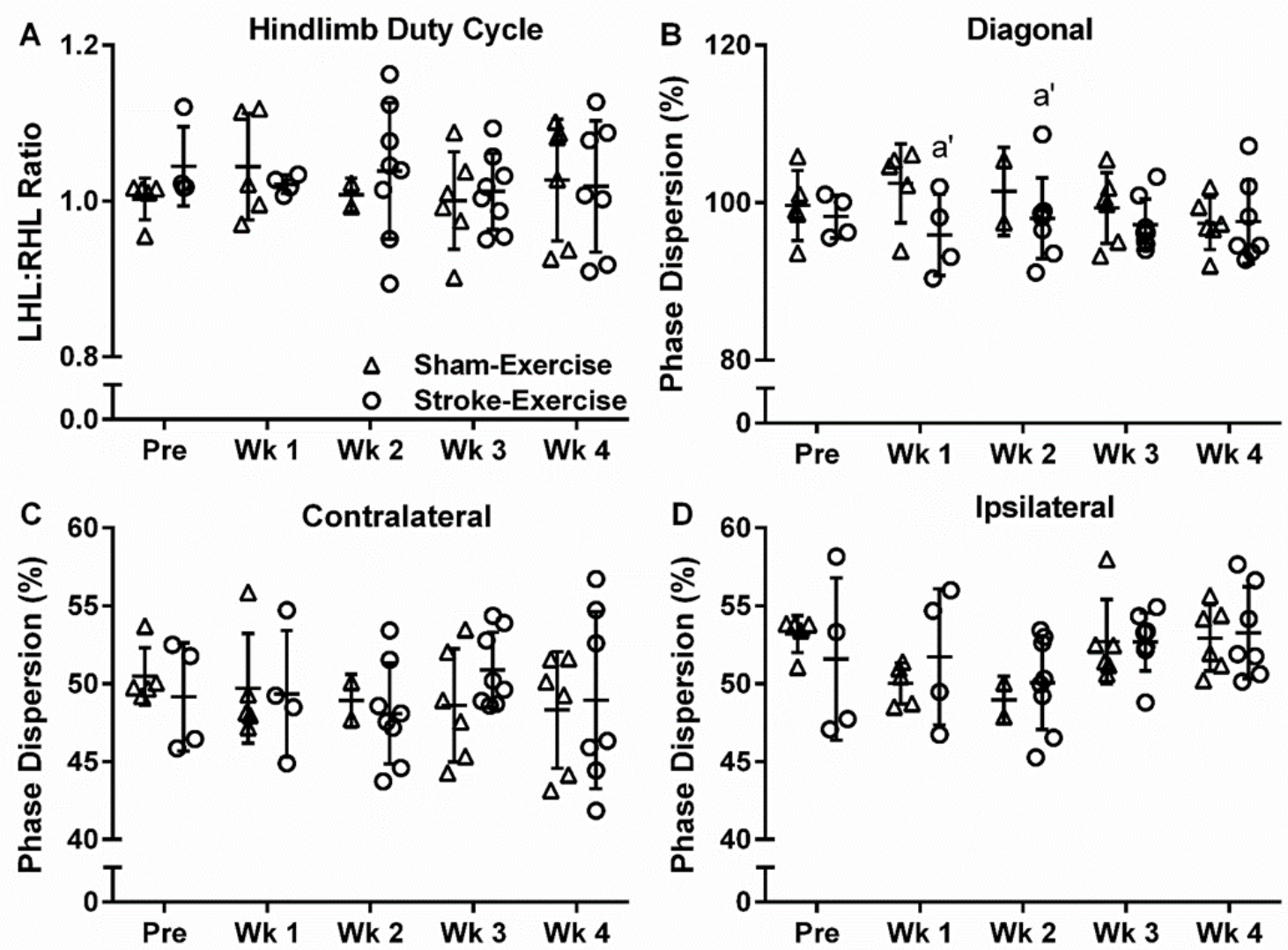
Figure 5
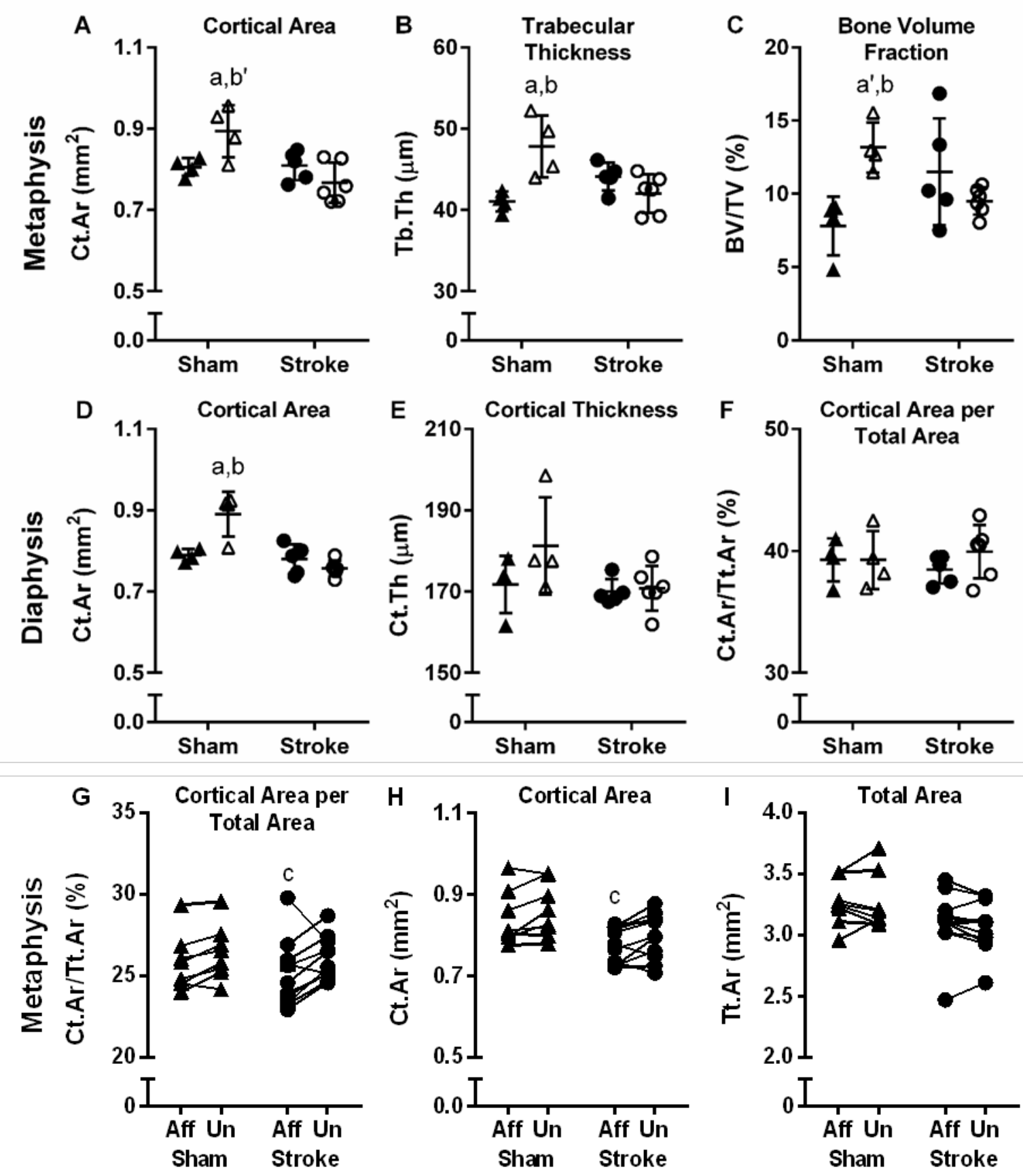
Figure 6
$\Delta$ Sham-Sedentary
$\Delta$ Sham-Exercise
Stroke-Sedentary
O Stroke-Exercise

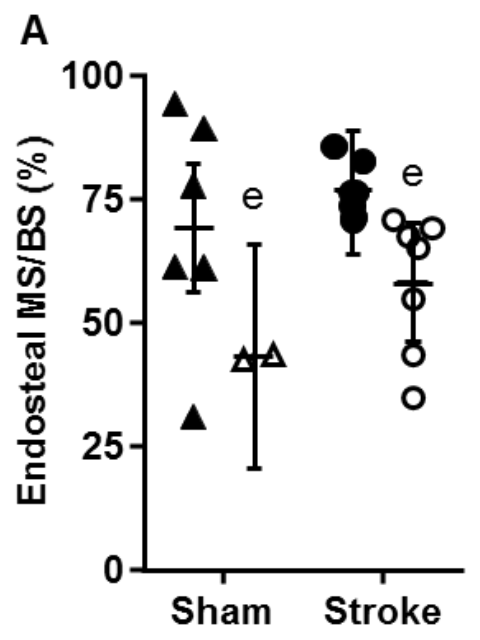

B

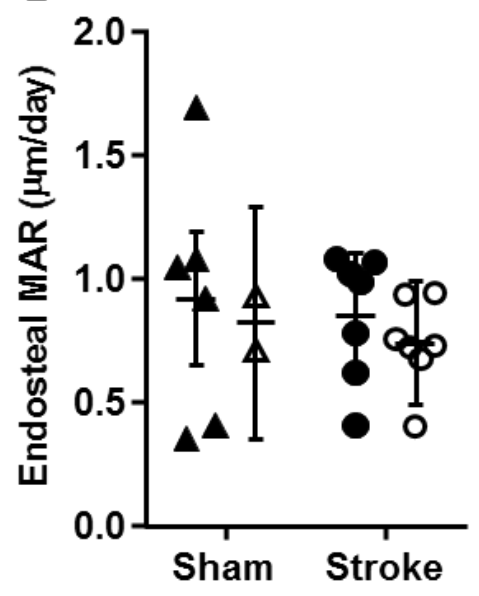

D

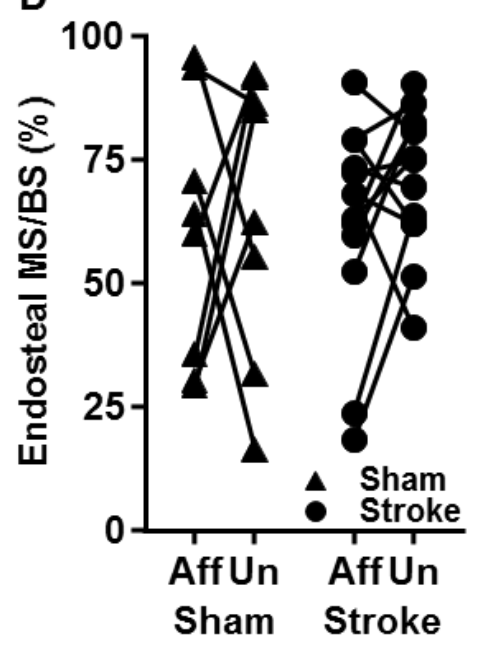

E

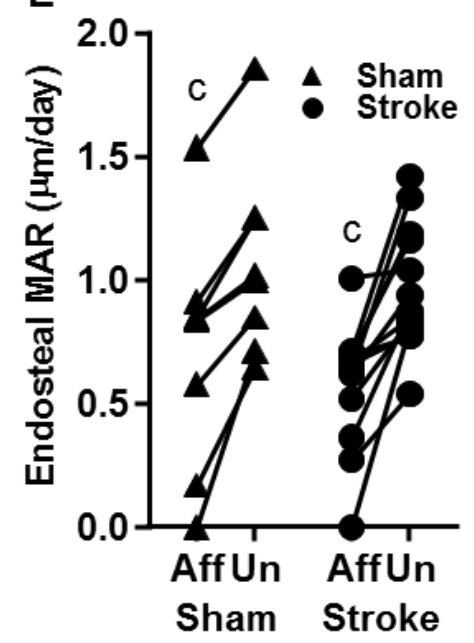

C
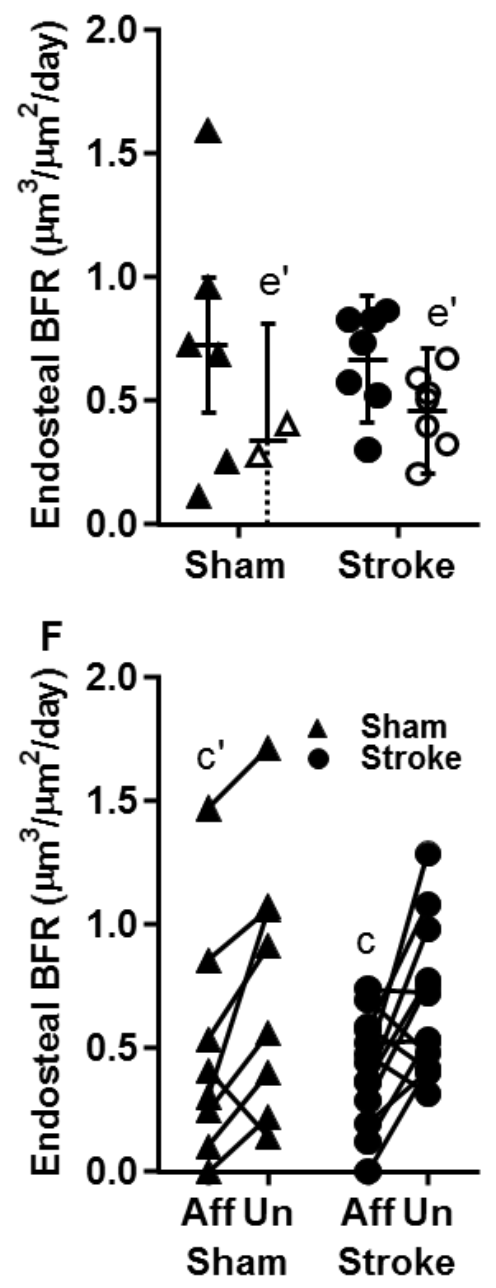
Figure 7
$\Delta$ Sham-Sedentary
$\Delta$ Sham-Exercise
- Stroke-Sedentary
O Stroke-Exercise

A

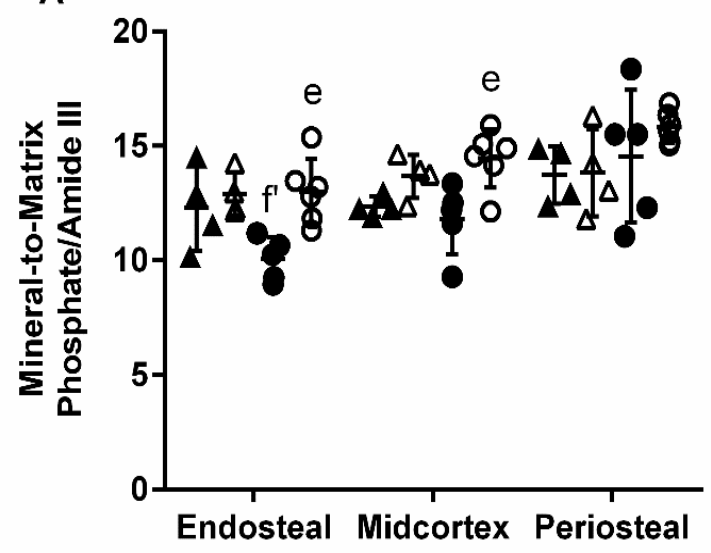

C

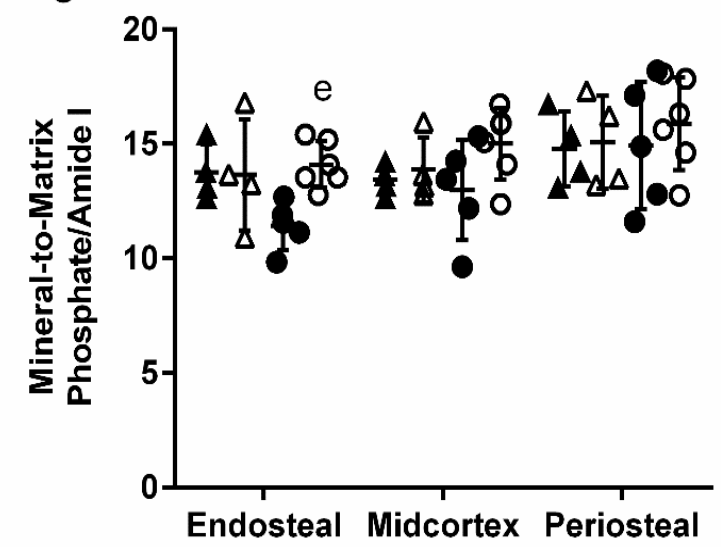

E

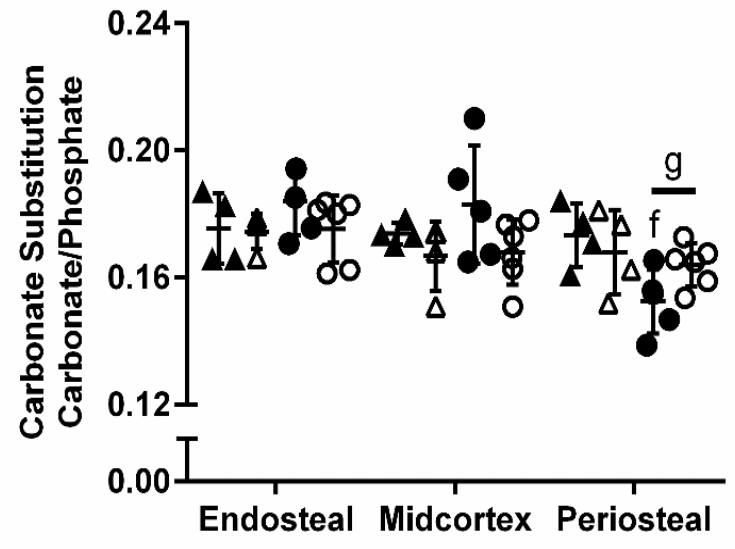

B

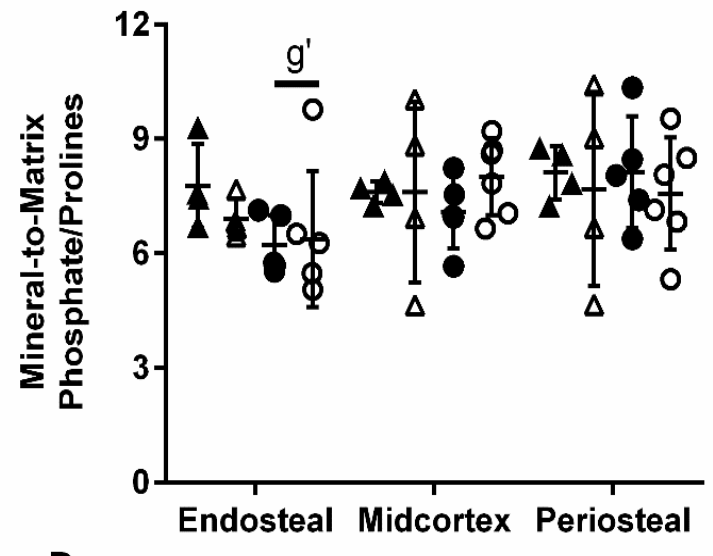

D
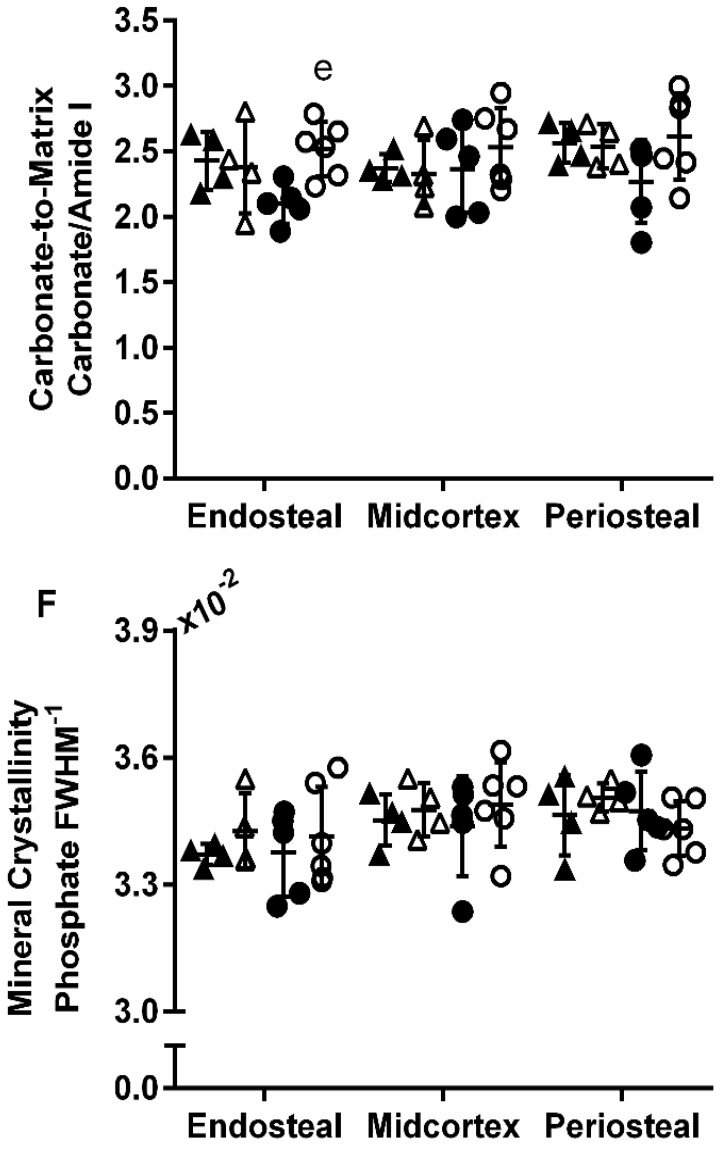